\title{
STUDY ON VARIABILITY AND GENETIC PARAMETERS IN ASH GOURD [BENINCASA HISPIDA (THUNB.) COGN] GENOTYPES
}

\author{
KATTULA NAGARAJU ${ }^{1} \&$ SARASWATHI T ${ }^{2}$ \\ ${ }^{I}$ Division of Vegetable Crops, Icar- Iihr, Bengaluru, Ka, India \\ ${ }^{2}$ Horticultural Research Station, Tnau, Kodaikanal Tamil Nadu, India
}

\begin{abstract}
Forty genotypes of ash gourd [Benincasa hispida (Thunb.) Cogn.] were evaluated to study their per se performance, genetic variability, heritability and genetic advance per cent of mean with the aim to screen potential genotypes for future improvement programmes. The genotypes were collected from various growing areas of India. Based on the per se performance, the non scented accessions viz., Coimbatore Local, IC 339164, KAG 15, PAG 72, IVAG 10, Indu, Chalkumar, KAU Local and Shadnagar Local and scented genotypes viz., IC 596991, IC 596992 and IC 596994 were identified as the best ones for yield and quality characters. The analysis of variance revealed significant variability among the genotypes for all the 18 traits during kharif, summer and pooled mean of both the seasons. Vine length, average fruit weight, flesh thickness, polar diameter of fruit, equatorial diameter of fruit, number of seeds per fruit, yield per vine, carbohydrate content and total soluble solids recorded high estimates of GCV, PCV, heritability and genetic advance as per cent of mean during both kharif and summer seasons and also in pooled analysis which indicate the probable likelihood of additive gene action. Thus, improving these characters should be effective and rewarding during selection.

KEYWORDS: Genetic Variability, Heritability, Genetic Advance as per Cent of Mean, Additive Gene Action, Selection Criteria
\end{abstract}

Received: Apr 08, 2016; Accepted: Apr 19, 2016; Published: Apr 22, 2016; Paper Id.: IJASRJUN2016019

\section{INTRODUCTION}

Ash gourd [Benincasa hispida (Thunb.) Cogn.] (Syn. Benincasa cerifera) is an important sub-tropical and tropical cucurbitaceous vegetable. The fruit is fuzzy when immature. By maturity, the fruit loses its hairs and develops a waxy coating, giving rise to the name 'wax gourd', and providing a long shelf life. Indo-China is the centre of diversity of ash gourd (Rubatazky and Yamaguchi, 1999). Its primary basic chromosome number is $\mathrm{x}=6$. Ash gourd is extensively grown in India for its nutritional, medicinal as well as curative properties. The fruits are consumed in various ways, as fresh vegetables, candied, dried, pickled, and also used in ayurvedic medicine preparation. Fruit of this plant are traditionally used to treat a renal diseases, urinary infection and biliousness (Nayar and More, 1998). The methanolic extract of the fruit is reported to possess anti-ulcer (Grover et al., 2001), anti-inflammatory (Chandrababu et al., 2002), antihistaminic, and antidepressant activities (Anilkumar et al., 2002). Fruits of Benincasa hispida are traditionally used for treatment of week nervousness and debility (Nadhiya et al., 2014). It has also great demand in processing industries for preparation of a popular sweet called 'petha'.

The presence and magnitude of genetic variation in a given gene pool is pre-requisite for selection of genotypes (Tiwari et al., 2011). Heritability of a trait is an important factor to be considered for genetic improvement of crops for 
quantitative traits as it determines response of the trait to selection. Genetic advance further provides information on expected genetic gain in the progeny resulting from selection of superior individuals. Yield per vine is a complex trait controlled by poly genes interacting with the environment and is the effect of the many factors called yield components. Selection of superior genotypes exhibiting high heritability and genetic advance for various characters is essential for improving the yield traits.

\section{MATERIALS AND METHODS}

The field experiment of the present study on "Diversity analysis in scented and non-scented ash gourd [Benincasa hispida. (Thunb.) Cogn.] Genotypes" was carried out at the College Orchard, Department of Vegetable Crops, Horticultural College and Research Institute, Tamil Nadu Agricultural University, Coimbatore during 2013-2014. The experiment was conducted for two seasons viz., kharif, 2013 (June - December) and summer, 2014 (January - June). Forty genotypes collected from different sources are used as biological materials for this study are furnished in Table 1. All the genotypes were raised in randomized block design (RBD) with three replications. Pits of $60 \mathrm{~cm}$ diameter and $30 \mathrm{~cm}$ depth were taken at a spacing of $1.5 \times 1.0 \mathrm{~m}^{\square}$. The forty genotypes (40 treatments) in each replication consist of ten pits and three seeds were sown in each pit. The cultural and management practices were adopted according to the package of practices recommended by Tamil Nadu Agricultural University.

Eighteen biometrical traits of ash gourd were recorded on five randomly selected plants in each of the accession per replication. The mean values were utilized for statistical analysis. ANOVA was worked out for all the characters by making use of means of replication, as suggested by Goulden (1959). Standard statistical procedures were adopted for calculating the mean, range and various genetic parameters like phenotypic coefficient of variation (PCV), genotypic coefficient of variation $(\mathrm{GCV})$, heritability $\left(\mathrm{h}^{2}\right)$ in broad sense and genetic advance as per cent of mean (GAM).

\section{RESULTS AND DISCUSSIONS}

The analysis of variance for 14 biometrical and four biochemical traits of ash gourd accessions raised during kharif and summer and their pooled variance are presented in Tables 2. The analysis of variance revealed significant differences among the genotypes for all the 18 traits during kharif, summer and pooled mean of both the seasons. The mean performance of the forty genotypes of ash gourd for all the characters studied is furnished in Tables 4 -12. Estimates on genotypic variance (GV), phenotypic variance (PV), Genotypic Co-efficient of variation (GCV), Phenotypic Co-efficient of variation (PCV), heritability $\left(\mathrm{h}^{2}\right)$ and genetic advance as per cent of mean (GAM) for eighteen different traits estimated during kharif, summer and pooled data are presented in Table 3.

\section{Per Se Performance}

Mean performance is the fundamental selection criterion for distinguishing superior and inferior performing genotypes. Based on the per se performance, the non scented accessions viz., Coimbatore Local, IC 339164, KAG 15, PAG 72, IVAG 10, Indu, Chalkumar, KAU Local and Shadnagar Local and scented genotypes viz., IC 596991, IC 596992 and IC 596994 were identified as the best ones for yield and quality characters.

\section{Variability Parameters}

Studies on genetic parameters such as PV, GV, PCV, GCV, heritability and GAM provide basic information regarding the genetic properties of the given gene pool, based on which breeding methods are to be formulated for further improvement of the crop. 
In the present investigation, the genotypes exhibited considerable amount of variability for all the 18 traits studied. Numerically, the phenotypic coefficient of variation (PCV) was higher than the genotypic coefficient of variation (GCV) for most of the characters indicating that these are having an interaction with the environment to some extent (Ram and Singh, 1993). Differences between the phenotypic and genotypic coefficients of variations were observed to be narrow for all the characters indicating the least influence of environment on the expression of these characters.

Vine length, sex ratio, average fruit weight, flesh thickness, polar diameter of fruits, equatorial diameter of fruits, number of seeds per fruit, yield per vine, carbohydrate content and total soluble solids recorded high genotypic and phenotypic coefficients of variation during Kharif and summer season as well as in pooled analysis.

In both seasons of evaluation, moderate GCV and PCV were recorded by internodal length, number of primary branches, node at first female flower appeared, hundred-seed weight, number of fruits per vine, crude fibre content and protein content whereas, days to first female flower opening registered low genotypic and phenotypic coefficients of variation.

Genotypic coefficient of variation alone does not give an idea of total variation that is due to genotypes. Further, it may not be feasible to determine the amount of heritable variation and the relative degree to which a character is transmitted from parent to offspring, by the estimate of heritability (Hanson, 1959). Heritability estimate in broad sense alone, does not serve as the true indicator of genetic potentiality of the genotype since the scope is restricted by their interaction with environment. Hence, it is advisable to consider the predicted genetic advance as per cent of mean along with heritability estimate as a reliable tool in selection programme (Johnson et al., 1955). Hence, both heritability and genetic advance as per cent of mean were determined to get a clear picture of the scope of improvement in various characters through selection.

Node at first female flower appeared, vine length, internodal length, sex ratio, average fruit weight, flesh thickness, polar diameter of fruits, equatorial diameter of fruits, number of seeds per fruit, yield per vine, carbohydrate content, protein content and total soluble solids recorded high heritability coupled with genetic advance as per cent of mean during kharif and summer seasons as well as in pooled analysis. This indicated that the variation due to environment played relatively limited role in influencing the inheritance of these characters and the heritability was due to additive genetic effects suggesting the response to selection would be higher.

High heritability coupled with moderate genetic advance as per cent of mean were recorded for days to first female flower opening and hundred-seed weight in kharif and summer seasons whereas hundred-seed weight recorded higher heritability and genetic advance as per cent of mean for this trait in pooled analysis. It can be concluded that high heritability and moderate genetic advance are indication of higher additive genetic effects in governing the traits.

Number of fruits per vine in both kharif and summer season, crude fibre content in kharif and number of primary branches in summer season recorded low heritability coupled with low genetic advance as per cent of mean.

Low GCV and PCV and high heritability and moderate genetic advance as per cent of mean were observed for days to first female flower opening during both kharif and summer seasons and also in pooled analysis. These results are in conformity with the findings of Sureja et al. (2010), Lovely et al. (2010) and Resmi and Sreelathakumary (2011) in ash gourd.

Gangopadhyaya et al. (2008) recorded moderate GCV and PCV and high heritability and genetic advance as per cent 
of mean for node at first female flower appeared, Resmi and Sreelathkumary (2011) for internodal length, Gangopadhyaya $\boldsymbol{e t}$ al. (2008), Lovely et al. (2010) and Resmi and Sreelathakumary (2011) for number of primary branches in ash gourd, Kumaran et al. (2000) in pumpkin and Resmi and Sreelathakumary (2011) in ash gourd for hundred-seed weight, Sureja et al. (2010) in ash gourd for crude fibre content, Sureja et al. (2010) in ash gourd and Kothainayagi (2013) in pumpkin for protein content.

In the present study, the heritability values were quite high for all the characters, indicating that the major part of the variability was due to genotypic causes. High heritability also indicated that there was more number of additive genes for these characters. The results are in line with the findings of Kumar et al. (2008) in cucumber and Hanchinamani $\boldsymbol{e t}$ al. (2011) in cucumber.

Vine length, average fruit weight, flesh thickness, polar diameter of fruit, equatorial diameter of fruit, number of seeds per fruit, yield per vine, carbohydrate content and total soluble solids recorded high estimates of GCV, PCV, heritability and genetic advance as per cent of mean during both kharif and summer seasons and also in pooled analysis. Similar results were obtained by Kumaran et al. (2000) in pumpkin and Resmi and Sreelathkumary (2011) in ash gourd for vine length; Singh et al. (2002), Sureja et al. (2010), Lovely et al. (2010) and Resmi and Sreelathakumary (2011) for yield per vine in ash gourd; Tamilselvi (2010) in pumpkin for carbohydrate content and Kumaran et al. (2000) and Muralidhara (2009) in pumpkin for total soluble solids.

\section{CONCLUSIONS}

From the present investigation it is concluded that. The considerable genetic variability was observed among the forty ash gourd genotypes. High heritability coupled with high GAM was recorded for vine length, average fruit weight, flesh thickness, polar diameter of fruit, equatorial diameter of fruit, number of seeds per fruit, yield per vine, carbohydrate content and total soluble solids.

\section{ACKNOWLEDGEMENT}

The Author would like to acknowledge the financial support of ICAR-JRF fellowship, Government of India during the M.Sc. Research Programme to conduct this experiment.

\section{REFERENCES}

1 Anilkumar, D. and Ramu, P. (2002). Effect of methanolic extract of Benincasa hispida against histamine and acetyl choline induced bronchospasm in guinea pigs. Indian J Pharmacol., 34: 365-6.

2 Chandrababu, S. and Umamaheshwari, S. (2002). Studies on the anti inflammatory activity of fruit rind extract of Benincasa hispida Cogn. Indian Drugs., 39: 651-3.

3 Gangopadhyay, K. K., Kumar, G, Meena B. L., and Bisht, I. S. (2008). Genetic diversity studies in ash gourd [Benincasa hispida (Thunb.) Cogn.] from northern India. J. Plant Genet. Resour. 21 (3): 206-212.

4 Goulden, C. H. (1952). Methods of statistical analysis. John Wiley and sons. Inc., New York.

5 Hanchinamani, C. N., Patil, M. G., Dharmati, P. R. and Mokashi, A. N. (2011). Studies on heritability and genetic advance in cucumber (Cucumis sativus L.). Crop. Res., 41 (1, 2 \& 3):160-163.

6 Hanson, W.D. (1959). Estimates of genetic parameters in cross-fertilizing plants and their implications in plant breeding. Statistical Genetics and Plant Breeding. NAS-NRC Publ. 982, pp 225-252. 
7 Johnson, H.W., Robinson, H.F. and Comstock, R.E. (1955). Estimation of genetic and environmental variability in soyabean. Agron. J., 41: 314-318.

8 Kumar, A., Kumar, S. and Pal, A.K. (2008). Genetic variability and character association for fruit yield and traits in cucumber. Indian J. Hort., 65 (4): 423-428.

9 Kumaran, S., Natarajan, S., and Thamburaj, S. (2000). Genetic variability in pumpkin (Cucurbita moschata Duch. Ex. Poir.). South Indian Hort., 45 (1\&2): 10-12.

10 Lovely, B. (2010). Estimates of genetic parameters in ash gourd [Benincasa hispida (Thunb.) Cogn.] M.Sc. (Hort.) Thesis, Kerala Agricultural University, Thrissur.

11 Muralidhara, M.S. (2009). Genetic variability studies in pumpkin (Cucurbita moschata Duch. Ex. Poir). M.Sc.(Hort.) Thesis, University of Agriculture Sciences, Bangalore.

12 Nadhiya, K., Haripriya, D. and Vijayalakshmi, K. (2014). Pharmacognostic and preliminary phytochemical analysis on Benincasa hispida fruit. Asian J. Pharm Clin Res., 7 (1):98-101.

13 Nayar, N. M. and More, T.A. (1998). Cucurbits. Oxford \& IBH Publishing Co. Pvt. Ltd., New Delhi. pp. 340.

14 Resmi J. and Sreelathakumary, I. (2011). Genetic variability and character associations in ash gourd [Benincasa hispida (Thunb.) Cogn.]. Agric. Sci. Digest., 31 (3): 193 - 197.

15 Rubatazky, V.E., and Yamaguchi, M. (1999). World Vegetables, Chapman and Hall, New York, USA pp. 843

16 Sureja, A.K., Sirohi, P.S., Patel, V.B. and Mahure, H.R. (2010). Estimation of genetic parameters in ash gourd. Indian J. Hort. 67: 170-173

17 Tamilselvi, N. A. (2010). Studies on heterosis and combining ability in pumpkin (Cucurbita moschata Duch. ex Poir). M.Sc., (Hort.) Thesis submitted to Tamil Nadu Agricultural University, Coimbatore, India.

18 Tiwari, R, Suresh, B. G., Mishra, V. K., Kumar A. and Kumar A. (2011). Genetic variability and character association in direct seeded upland rice (Oryza sativa). mitogen-activated protein kinase kinases Publication 29 (4A): 2132-2135.

\section{APPENDICES}

Table 1: List of Accessions Collected and Their Source

\begin{tabular}{|c|c|c|c|c|}
\hline S. No. & $\begin{array}{l}\text { Accession } \\
\text { No. }\end{array}$ & Accession Name & Source & $\begin{array}{l}\text { Scented / Non } \\
\text { scented Type }\end{array}$ \\
\hline 1 & $\mathrm{Bh} 1$ & BH 1 & TNAU, Coimbatore & Non- scented \\
\hline 2 & $\mathrm{Bh} 2$ & $\mathrm{BH} 2$ & TNAU, Coimbatore & Non- scented \\
\hline 3 & $\mathrm{Bh} 3$ & BH 3 & TNAU, Coimbatore & Non- scented \\
\hline 4 & Bh 4 & Chalkumar & TNAU, Coimbatore & Non- scented \\
\hline 5 & Bh 5 & Chityal Local & Chityal, Andhra Pradesh & Non- scented \\
\hline 6 & Bh 6 & $\mathrm{CO} 2$ & TNAU, Coimbatore & Non- scented \\
\hline 7 & Bh 7 & Coimbatore Local & TNAU, Coimbatore & Non- scented \\
\hline 8 & Bh 8 & DAG 6 & IARI, New Delhi & Non- scented \\
\hline 9 & Bh 9 & Debpurna & BCKV, West Bengal & Non- scented \\
\hline 10 & Bh 10 & Haveri Local & Haveri, Karnataka & Non- scented \\
\hline 11 & Bh 11 & HYBH 25 & TNAU, Coimbatore & Non- scented \\
\hline 12 & Bh 12 & IC 339164 & NBPGR, New Delhi & Non- scented \\
\hline 13 & Bh 13 & IC 339178 & NBPGR, New Delhi & Non- scented \\
\hline 14 & Bh 14 & IC 339180 & NBPGR, New Delhi & Non- scented \\
\hline 15 & Bh 15 & IC 339195 & NBPGR, New Delhi & Non- scented \\
\hline 16 & Bh 16 & Indu & KAU, Thrissur, Kerala & Non- scented \\
\hline 17 & Bh 17 & Indu-K & KAU, Thrissur, Kerala & Non- scented \\
\hline
\end{tabular}




\begin{tabular}{|l|l|l|l|l|}
\hline 18 & Bh 18 & IVAG 10 & IIVR, Varanasi & Non- scented \\
\hline 19 & Bh 19 & IVAG 3 & IIVR, Varanasi & Non- scented \\
\hline 20 & Bh 20 & IVAG 90 & IIVR, Varanasi & Non- scented \\
\hline 21 & Bh 21 & KAG 1 & KAU, Kerala & Non- scented \\
\hline 22 & Bh 22 & KAG 15 & KAU, Kerala & Non- scented \\
\hline 23 & Bh 23 & KAG 3 & KAU, Kerala & Non- scented \\
\hline 24 & Bh 24 & Kashi Surabhi & IIVR, Varanasi & Non- scented \\
\hline 25 & Bh 25 & Kashi Ujwal & IIVR, Varanasi & Non- scented \\
\hline 26 & Bh 26 & Kashi Dhawal & IIVR, Varanasi & Non- scented \\
\hline 27 & Bh 27 & KAU local & KAU, Thrissur, Kerala & Non- scented \\
\hline 28 & Bh 28 & Large fruited & KAU, Thrissur, Kerala & Non- scented \\
\hline 29 & Bh 29 & PAG 3 & TNAU, Coimbatore & Non- scented \\
\hline 30 & Bh 30 & PAG 72 & TNAU, Coimbatore & Non- scented \\
\hline 31 & Bh 31 & Pusa Ujwal & NBPGR, New Delhi & Non- scented \\
\hline 32 & Bh 32 & RCAG 15 & $\begin{array}{l}\text { ICAR Research complex } \\
\text { for NEH region, } \\
\text { Arunachal Pradesh }\end{array}$ & Non- scented \\
\hline 33 & Bh 33 & RCAG 28 & $\begin{array}{l}\text { ICAR Research complex } \\
\text { for NEH region, } \\
\text { Arunachal Pradesh }\end{array}$ & Non- scented \\
\hline 34 & Bh 34 & Shadnagar Local & $\begin{array}{l}\text { Shadnagar, Andhra } \\
\text { Pradesh }\end{array}$ & Non- scented \\
\hline 35 & Bh 35 & Vaidyakumbalam & KAU, Thrissur, Kerala & Non- scented \\
\hline 36 & Bh 36 & Varanasi Local & IIVR, Varanasi & Non- scented \\
\hline 37 & Bh 37 & IC 596991 & NBPGR, New Delhi & Scented \\
\hline 38 & Bh 38 & IC 596992 & NBPGR, New Delhi & Scented \\
\hline 39 & Bh 39 & IC 596993 & NBPGR, New Delhi & Scented \\
\hline 40 & Bh 40 & IC 596994 & NBPGR, New Delhi & Scented \\
\hline & & & & \\
\hline
\end{tabular}

Table 2: Analysis of Variance for Eighteen Characters in Ash Gourd During kharif, Summer Seasons and Pooled Data of Both the Seasons

\begin{tabular}{|c|c|c|c|c|c|c|c|c|c|}
\hline \multirow{3}{*}{ Characters } & \multicolumn{3}{|c|}{ kharif Season } & \multirow{2}{*}{\multicolumn{3}{|c|}{ Summer Season }} & \multirow{2}{*}{\multicolumn{3}{|c|}{$\begin{array}{c}\text { Pooled Data of } \text { Kharif and Summer } \\
\text { Seasons } \\
\text { Mean Sum of Squares }\end{array}$}} \\
\hline & Me: & Sum of Squ & & & & & & & \\
\hline & Replication & Treatment & Error & Replication & Treatment & Error & Replication & $\begin{array}{c}\text { Treatm } \\
\text { ent }\end{array}$ & Error \\
\hline Vine length & 96.97 & $\begin{array}{c}542478.20 \\
* *\end{array}$ & 1728.63 & 40061.9 & $496402.10^{* *}$ & 70921.62 & 11005.2 & $\begin{array}{c}516164 . \\
70 * *\end{array}$ & $\begin{array}{c}20208 \\
.14 \\
\end{array}$ \\
\hline Inter nodal length & 0.01 & $186.35^{* *}$ & 27.57 & 26.13 & $206.97 * *$ & 46.24 & 6.73 & $\begin{array}{c}189.39 * \\
*\end{array}$ & 22.52 \\
\hline $\begin{array}{l}\text { Number of } \\
\text { primary branches }\end{array}$ & 0.01 & $10.55 * *$ & 0.49 & 4.70 & $6.39 * *$ & 2.37 & 1.19 & $7.90 * *$ & 1.00 \\
\hline $\begin{array}{l}\text { Days to first } \\
\text { female flower } \\
\text { opening }\end{array}$ & 0.21 & $4118.64 * *$ & 247.87 & 31.17 & $3571.48 * *$ & 115.12 & 6.68 & $\begin{array}{l}3827.08 \\
* *\end{array}$ & 140.8 \\
\hline $\begin{array}{l}\text { Node at first } \\
\text { female flower } \\
\text { appeared }\end{array}$ & 0.5 & $1050.34 * *$ & 204.78 & 0.22 & $1081.27 * *$ & 110.48 & 0.34 & $\begin{array}{c}1060.07 \\
* *\end{array}$ & $\begin{array}{c}145.9 \\
9\end{array}$ \\
\hline Sex ratio & 0.26 & $46.52 * *$ & 3.74 & 1.74 & $101.53 * *$ & 16.68 & 0.50 & $70.94 * *$ & 3.19 \\
\hline $\begin{array}{l}\text { Average fruit } \\
\text { weight }\end{array}$ & 0.06 & $190.95 * *$ & 7.01 & 0.53 & $190.76^{* * *}$ & 9.42 & 0.14 & $\begin{array}{c}190.33^{*} \\
*\end{array}$ & 7.77 \\
\hline Flesh thickness & 1.00 & $155.51 * *$ & 10.75 & 0.41 & $154.85 * *$ & 13.07 & 0.58 & $\begin{array}{c}154.79 * \\
*\end{array}$ & 11.27 \\
\hline $\begin{array}{l}\text { Polar diameter of } \\
\text { fruit }\end{array}$ & 32.94 & $1828.24 * *$ & 154.03 & 59.71 & $2006.54 * *$ & 164.76 & 39.85 & $\begin{array}{c}1914.68 \\
* *\end{array}$ & $\begin{array}{c}157.3 \\
4\end{array}$ \\
\hline $\begin{array}{l}\text { Equatorial } \\
\text { diameter of fruit }\end{array}$ & 16.64 & $1236.09 * *$ & 83.15 & 17.85 & $1401.35 * *$ & 123.03 & 2.36 & $\begin{array}{c}1312.80 \\
* *\end{array}$ & 93.29 \\
\hline $\begin{array}{l}\text { Number of seeds } \\
\text { per fruit }\end{array}$ & 278.03 & $\begin{array}{c}5065139.0 \\
0 * * \\
\end{array}$ & 9710.73 & 682.24 & $\begin{array}{c}5860463.00 * \\
* \\
\end{array}$ & 46485.29 & 15.2 & $\begin{array}{c}5453988 \\
.00 * * \\
\end{array}$ & $\begin{array}{c}18200 \\
.80 \\
\end{array}$ \\
\hline $\begin{array}{l}\text { Hundred seed } \\
\text { weight }\end{array}$ & 0.23 & $127.38 * *$ & 41.33 & 1.48 & $139.93 * *$ & 49.16 & 0.69 & $\begin{array}{c}133.13 * \\
*\end{array}$ & 11.53 \\
\hline $\begin{array}{l}\text { Number of fruits } \\
\text { per vine }\end{array}$ & 1.61 & $20.83 * *$ & 9.38 & 0.81 & $36.00 * *$ & 19.33 & 0.59 & $24.82^{* *}$ & 6.65 \\
\hline
\end{tabular}




\begin{tabular}{|c|c|c|c|c|c|c|c|c|c|}
\hline $\begin{array}{l}\text { Carbohydrate } \\
\text { content }\end{array}$ & 0.21 & $129.42 * *$ & 8.23 & 0.22 & $137.41 * *$ & 9.25 & 0.05 & $\begin{array}{c}133.48 * \\
*\end{array}$ & 8.73 \\
\hline $\begin{array}{l}\text { Crude fibre } \\
\text { content }\end{array}$ & 0.01 & $0.54 * *$ & 0.2 & 0.01 & $0.93 * *$ & 0.13 & 0.01 & $0.65^{* *}$ & 0.07 \\
\hline Protein content & 3416.13 & $\begin{array}{c}245094.70 \\
* *\end{array}$ & 9356.3 & 3060.79 & $260630.30^{* *}$ & 65758.62 & 3003.60 & $\begin{array}{c}237844 . \\
70^{* *}\end{array}$ & $\begin{array}{c}20836 \\
.73\end{array}$ \\
\hline $\begin{array}{l}\text { Total Soluble } \\
\text { Solids }\end{array}$ & 0.77 & $213.19 * *$ & 9.67 & 2.40 & $192.43 * *$ & 13.68 & 1.11 & $\begin{array}{c}201.41 * \\
*\end{array}$ & 8.85 \\
\hline Yield per vine & 0.18 & $1062.17 * *$ & 10.1 & 0.14 & $1697.74 * *$ & 7.08 & 0.12 & $\begin{array}{c}1354.41 \\
* *\end{array}$ & 5.08 \\
\hline
\end{tabular}

*Significant at $5 \%$ level **Significant at $1 \%$ level

Table 3: Genetic Parameters for 18 Characters in Ash Gourd in During kharif, Summer Seasons and Pooled Data of Both the Seasons

\begin{tabular}{|c|c|c|c|c|c|c|c|c|c|c|c|c|c|c|c|c|c|c|}
\hline \multirow[b]{2}{*}{ Characters } & \multicolumn{6}{|c|}{ kharif season } & \multicolumn{6}{|c|}{ summer season } & \multicolumn{6}{|c|}{ pooled data of kharif and summer seasons } \\
\hline & GV & PV & $\begin{array}{l}\mathrm{GCT} \\
(\%)\end{array}$ & $\begin{array}{l}\mathrm{PCV} \\
(\%)\end{array}$ & $\begin{array}{l}\mathrm{h}^{2} \\
(\%)\end{array}$ & GAll & GV & PV & $\begin{array}{l}G C V \\
(\%)\end{array}$ & $\begin{array}{l}\mathrm{PCV} \\
(\%)\end{array}$ & $\begin{array}{c}\mathrm{h}^{2} \\
(\%)\end{array}$ & GAIL & GV & PV & $\begin{array}{l}\mathrm{GCV} \\
(\%)\end{array}$ & $\begin{array}{l}\mathrm{PCV} \\
(\%)\end{array}$ & $\begin{array}{c}h^{2} \\
(\%)\end{array}$ & GAll \\
\hline $\mathrm{VL}$ & 4629.18 & 4651.34 & 22.3 & 22.35 & 99.52 & 45.82 & 39.67 & 4848.92 & 20.77 & 23.05 & 81.20 & 38.58 & 4325.31 & 4584.38 & 21.66 & 22.30 & 94.35 & 35 \\
\hline INL & 1.47 & 1.82 & 12.1 & 13.47 & 80.67 & 22.38 & 1.57 & 2.61 & 12.61 & 14.80 & 72.60 & 22.14 & 1.52 & 1.81 & 12.35 & 13.47 & 84.06 & 23.33 \\
\hline $\mathrm{NPB}$ & 0.08 & 0.09 & 18.32 & 18.96 & 0.93 & 36.46 & 0.05 & 0.075 & 13.37 & 17.34 & 59.50 & 21.23 & 0.06 & 0.08 & 15.7 & 17.22 & 83.07 & 29.47 \\
\hline DFFO & 14 & 37.32 & 8.79 & 9.19 & 91.49 & 17.32 & 30.03 & 31.51 & 8.10 & 8.29 & 95.30 & 29 & 2 & 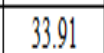 & 8.45 & 8.68 & 94.68 & 16.94 \\
\hline NFFA & 8.10 & 10.72 & 16.23 & 18.68 & 75.53 & 29.07 & 8.77 & 10.19 & 16.95 & 18.26 & 86.10 & 32.39 & 8.44 & 10.31 & 16.59 & 18.34 & 81.84 & 30.93 \\
\hline SR & 0.38 & 0.42 & 23.38 & 24.81 & 88.84 & 45.40 & 0.80 & 1.01 & 22.54 & 25.38 & 78.80 & 41.22 & 0.59 & 0.63 & 23.31 & 24.1 & 93.54 & 46.43 \\
\hline AFW & 1.60 & 1.69 & 46.35 & 47.63 & 94.69 & 92.92 & 1.59 & 1.71 & 44.75 & 46.42 & 92.90 & 88.88 & 1.59 & 1.69 & 45.51 & 46.91 & 94.11 & 90.94 \\
\hline $\mathrm{ET}$ & 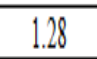 & 1.42 & 32.28 & 33.96 & 90.30 & 63.19 & 1.27 & 1.44 & 30.76 & 32.73 & 88.30 & 59.55 & 1.26 & 1.42 & 31.5 & 33.23 & 89.82 & 61.49 \\
\hline PD & 14.96 & 16.94 & 21.08 & 22.42 & 88.34 & 40.81 & 16.45 & 18.56 & 21.42 & 22.76 & 88.60 & 41.50 & 15.69 & 17.71 & 21.25 & 22.57 & 88.61 & 41.21 \\
\hline ED & 10.21 & 11.27 & 20.68 & 21.73 & 90.55 & 40.54 & 11.45 & 13.03 & 20.83 & 22.22 & 87.90 & 40.23 & 10.82 & 12.02 & 20.76 & 21.87 & 90.05 & 40.58 \\
\hline NSPF & 43250.30 & 43374.80 & 42.2 & 42.26 & 99.71 & 86.81 & 49890.8 & 50486.7 & 42.31 & 42.56 & 98.86 & 86.64 & 46537.50 & 46770.84 & 42.27 & 42.38 & 99.5 & 86.86 \\
\hline HSW & 0.91 & 1.44 & 11.59 & 14.57 & 63.25 & 18.98 & 0.99 & 1.62 & 11.42 & 14.02 & 61.00 & 18.37 & 1.09 & 1.24 & 12.32 & 13.13 & 88.04 & 23.81 \\
\hline NEPV & 0.15 & 0.258 & 14.96 & 20.47 & 53.42 & 22.52 & 0.23 & 0.47 & 15.81 & 22.92 & 47.60 & 22.47 & 0.18 & 0.27 & 15.65 & 18.93 & 68.30 & 26.64 \\
\hline YPV & 9.04 & 9.16 & 51.77 & 52.13 & 98.59 & 105.89 & 14.48 & 14.57 & 50.94 & 51.09 & 99.40 & 104.60 & 11.55 & 11.62 & 51.19 & 51.33 & 99.44 & 105.2 \\
\hline $\mathrm{CHO}$ & 1.07 & 1.18 & 25.02 & 26.23 & 91.03 & 49.18 & 1.13 & 1.25 & 25.04 & 26.32 & 90.50 & 49.08 & 1.11 & 1.22 & 25.02 & 26.26 & 90.79 & 49.12 \\
\hline $\mathrm{CF}$ & 0.0037 & 0.0063 & 12.97 & 16.77 & 59.78 & 20.66 & 0.07 & 0.09 & 18.16 & 20.15 & 81.20 & 33.70 & 0.005 & 0.006 & 15.22 & 16.56 & 84.52 & 28.83 \\
\hline PRO & 2054.84 & 2174.75 & 14.29 & 14.7 & 94.48 & 28.62 & 1946.59 & 2789.65 & 13.82 & 16.54 & 69.80 & 23.78 & 1943.82 & 2210.95 & 13.85 & 14.78 & 87.92 & 26.76 \\
\hline ISS & 1.78 & 1.9 & 52.48 & 54.28 & 93.49 & 104.54 & 1.59 & 1.76 & 47.25 & 49.78 & 90.00 & 92,35 & 1.68 & 1.79 & 49.83 & 51.48 & 93.69 & 99.35 \\
\hline
\end{tabular}

\section{Whereas,}

\begin{tabular}{|l|l|l|l|l|l|}
\hline VL & Vine length & AFW & Average fruit weight & NFPV & Number of fruits per vine \\
\hline INL & Internodal length & FT & Flesh thickness & YPV & Yield per vine \\
\hline NPB & $\begin{array}{l}\text { Number of primary } \\
\text { branches }\end{array}$ & PD & Polar diameter of fruit & CHO & Carbohydrate content \\
\hline DFFO & $\begin{array}{l}\text { Days to first female flower } \\
\text { opening }\end{array}$ & ED & Equatorial diameter of fruit & CF & Crude fiber content \\
\hline NFFA & $\begin{array}{l}\text { Node at first female flower } \\
\text { appeared }\end{array}$ & NSPF & Number of seeds per fruit & PRO & Protein content \\
\hline SR & Sex ratio & HSW & Hundred seed weight & TSS & Total soluble solids \\
\hline
\end{tabular}


Table 4: PER se Performance of Ash Gourd Genotypes for Vine Length and Internodal Length during Kharif, Summer and Pooled Data

\begin{tabular}{|c|c|c|c|c|c|c|}
\hline & \multicolumn{3}{|c|}{ Vine Length (cm) } & \multicolumn{3}{|c|}{ Internodal Length $(\mathrm{cm})$} \\
\hline Acc. No. & Kharif & Summer & Pooled & Kharif & Summer & Pooled \\
\hline $\mathrm{Bh} 1$ & 375.11 & 342.15 & 358.63 & 11.25 & 11.05 & 11.15 \\
\hline $\mathrm{Bh} 2$ & 315.02 & 315.23 & 315.12 & 10.48 & 10.17 & 10.33 \\
\hline $\mathrm{Bh} 3$ & 273.72 & 273.42 & 273.57 & 9.46 & 9.52 & 9.49 \\
\hline $\mathrm{Bh} 4$ & 291.85 & 296.72 & 294.28 & 10.53 & 10.39 & 10.46 \\
\hline Bh 5 & 313.59 & 302.17 & 307.89 & 10.81 & 10.68 & 10.75 \\
\hline Bh 6 & 328.98 & 339.83 & 334.4 & 10.81 & 10.54 & 10.67 \\
\hline $\mathrm{Bh} 7$ & 377.89 & 366.69 & 372.29 & 11.53 & 10.91 & 11.22 \\
\hline $\mathrm{Bh} 8$ & 343.13 & 336.57 & 339.85 & 10.18 & 10.12 & 10.15 \\
\hline Bh 9 & 345.69 & 370.89 & 358.29 & 11.04 & 10.86 & 10.95 \\
\hline Bh 10 & 392.27 & 382.14 & 387.2 & 10.81 & 10.53 & 10.67 \\
\hline Bh 11 & 303.42 & 301.54 & 302.48 & 10.01 & 10.14 & 10.08 \\
\hline Bh 12 & 406.46 & 400.83 & 403.65 & 12.08 & 11.88 & 11.98 \\
\hline Bh 13 & 365.85 & 360.94 & 363.39 & 9.89 & 9.89 & 9.89 \\
\hline Bh 14 & 343.36 & 339.70 & 341.53 & 9.97 & 9.88 & 9.93 \\
\hline Bh 15 & 260.78 & 262.56 & 261.67 & 10.28 & 10.18 & 10.23 \\
\hline Bh 16 & 315.62 & 329.40 & 322.51 & 10.69 & 10.51 & 10.6 \\
\hline Bh 17 & 283.76 & 272.28 & 278.02 & 10.74 & 10.47 & 10.61 \\
\hline Bh 18 & 342.84 & 348.88 & 345.86 & 9.64 & 9.34 & 9.50 \\
\hline Bh 19 & 292.17 & 284.72 & 288.44 & 10.71 & 10.49 & 10.6 \\
\hline Bh 20 & 347.19 & 332.22 & 339.7 & 10.62 & 10.27 & 10.45 \\
\hline $\mathrm{Bh} 21$ & 229.11 & 230.12 & 229.61 & 8.77 & 8.73 & 8.75 \\
\hline $\mathrm{Bh} 22$ & 378.67 & 365.56 & 372.11 & 9.96 & 9.87 & 9.92 \\
\hline Bh 23 & 239.25 & 233.13 & 236.19 & 10.02 & 9.97 & 10.00 \\
\hline $\mathrm{Bh} 24$ & 324.83 & 334.82 & 329.83 & 10.73 & 13.49 & 12.12 \\
\hline Bh 25 & 252.79 & 257.12 & 254.96 & 9.70 & 9.57 & 9.64 \\
\hline Bh 26 & 332.98 & 318.49 & 325.74 & 10.14 & 9.91 & 10.03 \\
\hline Bh 27 & 264.16 & 250.07 & 257.12 & 9.32 & 8.70 & 9.01 \\
\hline Bh 28 & 334.59 & 320.88 & 327.74 & 10.39 & 10.23 & 10.31 \\
\hline Bh 29 & 353.89 & 343.27 & 348.58 & 9.14 & 8.75 & 8.95 \\
\hline Bh 30 & 335.21 & 337.99 & 336.60 & 10.58 & 10.71 & 10.65 \\
\hline $\mathrm{Bh} 31$ & 340.67 & 335.11 & 337.89 & 10.55 & 10.39 & 10.47 \\
\hline Bh 32 & 294.37 & 292.35 & 293.36 & 9.08 & 8.87 & 8.98 \\
\hline Bh 33 & 303.65 & 304.36 & 304.01 & 11.17 & 10.86 & 11.02 \\
\hline Bh 34 & 303.57 & 296.35 & 299.96 & 10.99 & 10.62 & 10.81 \\
\hline Bh 35 & 396.67 & 386.36 & 391.51 & 11.18 & 10.9 & 11.04 \\
\hline Bh 36 & 295.58 & 286.03 & 290.81 & 9.66 & 9.52 & 9.59 \\
\hline Bh 37 & 174.43 & 174.91 & 174.68 & 7.27 & 7.18 & 7.23 \\
\hline Bh 38 & 97.66 & 98.41 & 98.04 & 5.13 & 5.13 & 5.13 \\
\hline Bh 39 & 127.08 & 134.36 & 130.72 & 7.65 & 7.84 & 7.75 \\
\hline $\mathrm{Bh} 40$ & 206.14 & 224.98 & 215.56 & 8.47 & 8.46 & 8.47 \\
\hline Mean & 305.10 & 302.09 & 303.60 & 10.04 & 9.94 & 9.99 \\
\hline Range & $406.46-97.66$ & $400.83-98.41$ & $403.65-98.04$ & $12.08-5.13$ & $13.49-5.13$ & $12.12-5.13$ \\
\hline $\mathbf{C V}$ & 1.54 & 9.98 & 5.30 & 5.92 & 7.74 & 5.37 \\
\hline SEd & 2.72 & 17.41 & 13.14 & 0.34 & 0.45 & 0.44 \\
\hline $\mathrm{CD}(\mathrm{P}=0.05)$ & 7.65 & 49.02 & 26.17 & 0.97 & 1.25 & 0.87 \\
\hline
\end{tabular}


Table 5: Per se performance of Ash Gourd Genotypes for Number of Primary Branches and Days to First Female Flower Opening during Kharif, summer and Pooled Data

\begin{tabular}{|c|c|c|c|c|c|c|}
\hline \multirow[b]{2}{*}{ Acc. No. } & \multicolumn{3}{|c|}{ Number of Primary Branches } & \multicolumn{3}{|c|}{ Days to First Female Flower Opening } \\
\hline & Kharif & Summer & Pooled & Kharif & Summer & Pooled \\
\hline Bh 1 & 2.26 & 2.18 & 2.23 & 67.33 & 68.53 & 67.93 \\
\hline Bh 2 & 1.32 & 1.33 & 1.32 & 75.75 & 77.03 & 76.39 \\
\hline Bh 3 & 1.40 & 1.40 & 1.41 & 66.33 & 68.43 & 67.38 \\
\hline Bh 4 & 1.45 & 1.53 & 1.49 & 66.58 & 67.67 & 67.13 \\
\hline Bh 5 & 1.38 & 1.40 & 1.39 & 72.58 & 72.9 & 72.74 \\
\hline Bh 6 & 1.50 & 1.49 & 1.50 & 62.08 & 63.06 & 62.58 \\
\hline Bh 7 & 1.47 & 1.54 & 1.51 & 61.95 & 64.80 & 63.37 \\
\hline Bh 8 & 1.53 & 1.55 & 1.54 & 62.53 & 63.08 & 62.81 \\
\hline Bh 9 & 1.33 & 1.39 & 1.36 & 76.14 & 76.76 & 76.45 \\
\hline Bh 10 & 1.39 & 1.39 & 1.39 & 75.17 & 75.32 & 75.24 \\
\hline Bh 11 & 1.50 & 1.43 & 1.47 & 62.17 & 63.7 & 62.93 \\
\hline Bh 12 & 1.63 & 1.68 & 1.66 & 57.06 & 58.63 & 57.85 \\
\hline Bh 13 & 1.46 & 1.49 & 1.48 & 56.83 & 59.73 & 58.28 \\
\hline Bh 14 & 1.34 & 1.39 & 1.36 & 57.83 & 59.93 & 58.88 \\
\hline Bh 15 & 1.43 & 1.37 & 1.4 & 60.28 & 61.44 & 60.86 \\
\hline Bh 16 & 1.75 & 1.62 & 1.69 & 59.75 & 61.66 & 60.71 \\
\hline Bh 17 & 1.59 & 1.57 & 1.58 & 62.39 & 64.18 & 63.29 \\
\hline Bh 18 & 1.61 & 1.69 & 1.65 & 65.70 & 65.75 & 65.72 \\
\hline Bh 19 & 1.70 & 1.71 & 1.70 & 63.25 & 64.53 & 63.89 \\
\hline Bh 20 & 2.00 & 1.94 & 1.97 & 66.78 & 67.54 & 67.16 \\
\hline Bh 21 & 1.61 & 1.68 & 1.65 & 55.88 & 57.43 & 56.66 \\
\hline Bh 22 & 2.08 & 2.10 & 2.09 & 65.22 & 66.33 & 65.78 \\
\hline Bh 23 & 1.66 & 1.70 & 1.68 & 72.03 & 73.20 & 72.61 \\
\hline Bh 24 & 1.57 & 1.55 & 1.56 & 65.75 & 66.81 & 66.28 \\
\hline Bh 25 & 1.49 & 1.41 & 1.45 & 69.25 & 70.6 & 69.92 \\
\hline Bh 26 & 1.39 & 1.39 & 1.39 & 61.25 & 61.81 & 61.53 \\
\hline Bh 27 & 1.61 & 1.69 & 1.65 & 60.82 & 64.23 & 62.53 \\
\hline Bh 28 & 1.49 & 1.39 & 1.44 & 71.86 & 72.80 & 72.33 \\
\hline Bh 29 & 1.80 & 1.46 & 1.64 & 66.11 & 66.68 & 66.4 \\
\hline Bh 30 & 1.93 & 1.92 & 1.93 & 64.56 & 65.74 & 65.15 \\
\hline Bh 31 & 1.37 & 1.38 & 1.38 & 72.28 & 73.53 & 72.9 \\
\hline Bh 32 & 1.42 & 1.51 & 1.47 & 67.17 & 67.83 & 67.5 \\
\hline Bh 33 & 2.65 & 2.13 & 2.39 & 62.22 & 63.39 & 62.81 \\
\hline Bh 34 & 1.50 & 1.42 & 1.46 & 75.83 & 75.19 & 75.51 \\
\hline Bh 35 & 1.34 & 1.36 & 1.35 & 72.31 & 73.22 & 72.77 \\
\hline Bh 36 & 1.45 & 1.47 & 1.46 & 63.45 & 64.87 & 64.16 \\
\hline Bh 37 & 2.32 & 2.09 & 2.20 & 75.86 & 76.39 & 76.13 \\
\hline Bh 38 & 1.59 & 1.44 & 1.52 & 71.58 & 72.29 & 71.94 \\
\hline Bh 39 & 2.00 & 1.49 & 1.75 & 72.64 & 73.94 & 73.29 \\
\hline Bh 40 & 1.48 & 1.49 & 1.49 & 74.08 & 74.97 & 74.53 \\
\hline Mean & 1.62 & 1.58 & 1.60 & 66.47 & 67.65 & 67.06 \\
\hline Range & $2.65-1.32$ & $2.18-1.33$ & $2.39-1.32$ & $76.14-55.88$ & $77.03-57.43$ & $76.45-56.66$ \\
\hline $\mathrm{CV}$ & 4.88 & 11.03 & 7.08 & 2.68 & 1.79 & 2.00 \\
\hline SEd & 0.05 & 1.01 & 0.09 & 1.03 & 0.7 & 1.09 \\
\hline $\mathrm{CD}(\mathrm{P}=0.05)$ & 0.13 & 0.28 & 0.18 & 2.89 & 1.97 & 2.18 \\
\hline
\end{tabular}


Table 6: Per se Performance of Ash Gourd Genotypes for Node at First Female Flower Appeared and Sex Ratio during Kharif, Summer and Pooled Data

\begin{tabular}{|c|c|c|c|c|c|c|}
\hline & \multicolumn{3}{|c|}{ Node at First Female Appeared } & \multicolumn{3}{|c|}{ Sex Ratio } \\
\hline Acc. No. & Kharif & Summer & Pooled & Kharif & Summer & Pooled \\
\hline Bh 1 & 21.57 & 22.79 & 22.18 & 2.55 & 3.66 & 3.11 \\
\hline Bh 2 & 15.97 & 15.74 & 15.86 & 2.35 & 3.49 & 2.92 \\
\hline Bh 3 & 16.33 & 17.05 & 16.7 & 2.45 & 3.81 & 3.13 \\
\hline Bh 4 & 19.83 & 20.02 & 19.93 & 2.33 & 3.37 & 2.85 \\
\hline Bh 5 & 20.1 & 20.32 & 20.21 & 3.47 & 5.02 & 4.25 \\
\hline Bh 6 & 19.03 & 18.91 & 18.98 & 2.52 & 3.80 & 3.16 \\
\hline Bh 7 & 18.2 & 18.11 & 18.15 & 2.52 & 3.64 & 3.08 \\
\hline Bh 8 & 19.37 & 19.03 & 19.2 & 2.10 & 3.18 & 2.64 \\
\hline Bh 9 & 18.13 & 18.47 & 18.3 & 2.19 & 3.40 & 2.80 \\
\hline Bh 10 & 18.67 & 18.83 & 18.75 & 2.14 & 3.19 & 2.67 \\
\hline Bh 11 & 22.3 & 22.15 & 22.23 & 2.58 & 3.74 & 3.16 \\
\hline Bh 12 & 19.87 & 20.02 & 19.95 & 2.81 & 3.92 & 3.37 \\
\hline Bh 13 & 17.9 & 17.61 & 17.76 & 2.76 & 4.07 & 3.41 \\
\hline Bh 14 & 14.00 & 13.50 & 13.75 & 2.42 & 3.64 & 3.03 \\
\hline Bh 15 & 12.43 & 12.36 & 12.4 & 2.46 & 3.61 & 3.03 \\
\hline Bh 16 & 18.97 & 18.97 & 18.97 & 2.53 & 3.90 & 3.22 \\
\hline Bh 17 & 17.83 & 18.87 & 18.35 & 2.42 & 3.92 & 3.17 \\
\hline Bh 18 & 20.07 & 19.66 & 19.87 & 2.20 & 3.31 & 2.76 \\
\hline Bh 19 & 23.27 & 22.65 & 22.96 & 2.23 & 3.39 & 2.81 \\
\hline Bh 20 & 19.43 & 19.42 & 19.43 & 2.48 & 3.70 & 3.09 \\
\hline Bh 21 & 14.97 & 14.70 & 14.83 & 2.66 & 3.84 & 3.25 \\
\hline Bh 22 & 18.73 & 19.22 & 18.98 & 2.00 & 3.02 & 2.51 \\
\hline Bh 23 & 19.70 & 19.58 & 19.64 & 2.21 & 3.64 & 2.93 \\
\hline Bh 24 & 17.80 & 17.00 & 17.4 & 2.47 & 3.95 & 3.22 \\
\hline Bh 25 & 17.27 & 16.89 & 17.08 & 2.27 & 3.56 & 2.92 \\
\hline Bh 26 & 18.10 & 17.93 & 18.02 & 2.41 & 3.63 & 3.02 \\
\hline Bh 27 & 19.67 & 19.47 & 19.57 & 2.28 & 3.62 & 2.95 \\
\hline Bh 28 & 16.60 & 16.84 & 16.72 & 2.49 & 3.54 & 3.02 \\
\hline Bh 29 & 13.27 & 14.11 & 13.69 & 5.36 & 8.11 & 6.74 \\
\hline Bh 30 & 19.43 & 18.75 & 19.09 & 2.20 & 3.27 & 2.74 \\
\hline Bh 31 & 22.00 & 21.52 & 21.76 & 3.55 & 5.34 & 4.45 \\
\hline Bh 32 & 18.43 & 18.16 & 18.3 & 4.45 & 6.50 & 5.48 \\
\hline Bh 33 & 16.97 & 16.39 & 16.68 & 2.49 & 3.72 & 3.11 \\
\hline Bh 34 & 13.93 & 13.39 & 13.66 & 2.99 & 4.54 & 3.77 \\
\hline Bh 35 & 16.57 & 16.51 & 16.54 & 2.95 & 4.62 & 3.78 \\
\hline Bh 36 & 16.13 & 16.17 & 16.15 & 2.74 & 4.10 & 3.42 \\
\hline Bh 37 & 11.23 & 11.03 & 11.14 & 2.22 & 3.31 & 2.77 \\
\hline Bh 38 & 13.57 & 13.49 & 13.53 & 2.62 & 3.78 & 3.20 \\
\hline Bh 39 & 11.87 & 11.67 & 11.77 & 3.03 & 4.54 & 3.78 \\
\hline Bh 40 & 11.73 & 11.55 & 11.64 & 2.75 & 4.06 & 3.40 \\
\hline Mean & 17.53 & 17.47 & 17.50 & 2.64 & 3.96 & 3.30 \\
\hline Range & $23.27-11.53$ & $22.79-11.03$ & $22.96-11.14$ & $5.36-2.00$ & $8.11-3.02$ & $6.74-2.51$ \\
\hline CV & 9.24 & 6.81 & 7.81 & 8.28 & 11.67 & 6.12 \\
\hline SEd & 0.96 & 0.69 & 1.12 & 0.13 & 0.27 & 0.17 \\
\hline $\mathrm{CD}(\mathrm{P}=0.05)$ & 2.63 & 1.93 & 2.22 & 0.36 & 0.75 & 0.33 \\
\hline
\end{tabular}


Table 7: Per Se Performance of Ash Gourd Genotypes for Average Fruit Weight and Flesh Thickness during Kharif, Summer and Pooled Data

\begin{tabular}{|c|c|c|c|c|c|c|}
\hline \multirow[b]{2}{*}{ Acc. No. } & \multicolumn{3}{|c|}{ Average Fruit Weight (kg) } & \multicolumn{3}{|c|}{ Flesh Thickness (cm) } \\
\hline & Kharif & Summer & Pooled & Kharif & Summer & Pooled \\
\hline $\mathrm{Bh} 1$ & 2.43 & 2.44 & 2.44 & 3.50 & 3.63 & 3.57 \\
\hline Bh 2 & 2.25 & 2.37 & 2.31 & 2.13 & 2.27 & 2.20 \\
\hline Bh 3 & 2.35 & 2.46 & 2.41 & 3.50 & 3.67 & 3.58 \\
\hline $\mathrm{Bh} 4$ & 3.25 & 3.41 & 3.33 & 2.30 & 2.43 & 2.37 \\
\hline Bh 5 & 3.72 & 3.81 & 3.77 & 5.90 & 6.07 & 5.98 \\
\hline Bh 6 & 3.29 & 3.35 & 3.32 & 3.93 & 4.00 & 3.97 \\
\hline $\mathrm{Bh} 7$ & 7.24 & 6.83 & 7.03 & 3.20 & 3.50 & 3.35 \\
\hline Bh 8 & 2.56 & 2.61 & 2.58 & 4.03 & 4.17 & 4.10 \\
\hline Bh 9 & 2.65 & 2.78 & 2.71 & 2.97 & 3.40 & 3.18 \\
\hline $\mathrm{Bh} 10$ & 2.72 & 2.75 & 2.73 & 5.00 & 5.10 & 5.05 \\
\hline $\mathrm{Bh} 11$ & 3.21 & 3.31 & 3.26 & 2.50 & 2.60 & 2.55 \\
\hline Bh 12 & 2.99 & 3.13 & 3.06 & 2.47 & 2.57 & 2.52 \\
\hline $\mathrm{Bh} 13$ & 1.19 & 1.26 & 1.23 & 2.80 & 2.93 & 2.87 \\
\hline $\mathrm{Bh} 14$ & 2.24 & 2.29 & 2.27 & 4.10 & 4.17 & 4.13 \\
\hline Bh 15 & 1.68 & 1.74 & 1.70 & 5.00 & 5.23 & 5.12 \\
\hline $\mathrm{Bh} 16$ & 4.66 & 4.87 & 4.77 & 4.03 & 4.23 & 4.13 \\
\hline Bh 17 & 2.17 & 2.25 & 2.21 & 3.93 & 3.07 & 4.00 \\
\hline Bh 18 & 2.68 & 2.8 & 2.74 & 3.13 & 3.30 & 3.22 \\
\hline Bh 19 & 5.23 & 5.38 & 5.31 & 3.13 & 3.23 & 3.18 \\
\hline $\mathrm{Bh} 20$ & 3.30 & 3.34 & 3.32 & 3.03 & 3.63 & 3.33 \\
\hline $\mathrm{Bh} 21$ & 1.96 & 2.05 & 2.01 & 3.03 & 3.17 & 3.10 \\
\hline $\mathrm{Bh} 22$ & 4.18 & 4.31 & 4.24 & 2.47 & 2.57 & 2.52 \\
\hline $\mathrm{Bh} 23$ & 2.45 & 2.57 & 2.51 & 4.00 & 4.20 & 4.10 \\
\hline $\mathrm{Bh} 24$ & 3.09 & 3.25 & 3.17 & 3.23 & 3.23 & 3.23 \\
\hline $\mathrm{Bh} 25$ & 3.07 & 3.11 & 3.09 & 5.37 & 5.47 & 5.42 \\
\hline Bh 26 & 2.34 & 2.44 & 2.39 & 3.03 & 3.17 & 3.10 \\
\hline Bh 27 & 2.89 & 2.90 & 2.89 & 2.77 & 2.77 & 2.77 \\
\hline $\mathrm{Bh} 28$ & 3.37 & 4.18 & 3.78 & 3.03 & 3.17 & 3.10 \\
\hline Bh 29 & 2.86 & 2.92 & 2.69 & 5.63 & 5.80 & 5.72 \\
\hline $\mathrm{Bh} 30$ & 3.56 & 3.69 & 3.62 & 2.67 & 2.77 & 2.72 \\
\hline $\mathrm{Bh} 31$ & 1.76 & 1.81 & 1.79 & 3.83 & 3.97 & 3.90 \\
\hline $\mathrm{Bh} 32$ & 2.87 & 2.90 & 2.89 & 5.17 & 5.27 & 5.22 \\
\hline $\mathrm{Bh} 33$ & 2.76 & 2.88 & 2.82 & 2.93 & 3.07 & 3.00 \\
\hline $\mathrm{Bh} 34$ & 3.93 & 3.99 & 3.96 & 6.27 & 6.40 & 6.33 \\
\hline Bh 35 & 1.69 & 1.75 & 1.72 & 4.90 & 5.07 & 4.98 \\
\hline $\mathrm{Bh} 36$ & 2.34 & 2.45 & 2.39 & 3.53 & 3.47 & 3.50 \\
\hline $\mathrm{Bh} 37$ & 0.65 & 0.66 & 0.65 & 1.97 & 2.00 & 1.98 \\
\hline Bh 38 & 0.44 & 0.45 & 0.45 & 2.20 & 2.57 & 2.38 \\
\hline Bh 39 & 0.76 & 0.77 & 0.76 & 1.73 & 1.93 & 1.83 \\
\hline $\mathrm{Bh} 40$ & 0.43 & 0.44 & 0.43 & 2.00 & 2.20 & 2.10 \\
\hline Mean & 2.73 & 2.82 & 2.77 & 3.51 & 3.66 & 3.59 \\
\hline Range & $7.24-0.43$ & $6.83-0.44$ & $7.03-0.43$ & $6.27-1.73$ & $6.40-1.93$ & $6.33-1.83$ \\
\hline $\mathrm{CV}$ & 10.97 & 12.33 & 11.38 & 10.57 & 11.18 & 10.60 \\
\hline SEd & 0.17 & 0.2 & 0.26 & 0.21 & 0.24 & 0.31 \\
\hline $\mathrm{CD}(\mathrm{P}=0.05)$ & 0.49 & 0.57 & 0.51 & 0.60 & 0.67 & 0.62 \\
\hline
\end{tabular}


Table 8: Per Se Performance of Ash Gourd Genotypes for Polar Diameter of Fruit and Equatorial Diameter of Fruit during kharif, Summer and Pooled Data

\begin{tabular}{|c|c|c|c|c|c|c|}
\hline \multirow[b]{2}{*}{ Acc. No. } & \multicolumn{3}{|c|}{ Polar Diameter $(\mathrm{cm})$} & \multicolumn{3}{|c|}{ Equatorial Diameter (cm) } \\
\hline & Kharif & Summer & Pooled & Kharif & Summer & Pooled \\
\hline $\mathrm{Bh} 1$ & 15.87 & 16.00 & 15.93 & 14.97 & 15.23 & 15.10 \\
\hline $\mathrm{Bh} 2$ & 19.37 & 20.30 & 19.83 & 15.00 & 15.97 & 15.48 \\
\hline $\mathrm{Bh} 3$ & 16.23 & 16.90 & 16.57 & 15.9 & 16.90 & 16.40 \\
\hline $\mathrm{Bh} 4$ & 18.23 & 19.17 & 18.70 & 18.77 & 19.90 & 19.33 \\
\hline $\mathrm{Bh} 5$ & 23.17 & 23.87 & 23.52 & 17.33 & 18.20 & 17.77 \\
\hline Bh 6 & 13.57 & 13.87 & 13.72 & 17.37 & 18.17 & 17.77 \\
\hline $\mathrm{Bh} 7$ & 26.00 & 26.97 & 26.48 & 24.63 & 24.70 & 24.67 \\
\hline $\mathrm{Bh} 8$ & 18.57 & 18.87 & 18.72 & 14.53 & 14.73 & 14.63 \\
\hline $\mathrm{Bh} 9$ & 26.33 & 27.40 & 26.87 & 16.63 & 17.90 & 17.27 \\
\hline Bh 10 & 23.57 & 23.87 & 23.72 & 15.10 & 15.57 & 15.33 \\
\hline Bh 11 & 19.03 & 19.33 & 19.18 & 17.30 & 18.70 & 18.00 \\
\hline Bh 12 & 24.57 & 25.43 & 25.00 & 18.37 & 19.97 & 19.17 \\
\hline Bh 13 & 17.50 & 18.30 & 17.90 & 11.83 & 13.00 & 12.42 \\
\hline Bh 14 & 14.80 & 15.13 & 14.97 & 18.50 & 19.33 & 18.92 \\
\hline Bh 15 & 19.77 & 20.37 & 20.07 & 14.33 & 15.23 & 14.78 \\
\hline Bh 16 & 21.83 & 22.80 & 22.32 & 17.20 & 18.10 & 17.65 \\
\hline Bh 17 & 17.50 & 18.13 & 17.82 & 17.67 & 18.93 & 18.3 \\
\hline Bh 18 & 20.17 & 21.13 & 20.65 & 16.43 & 17.67 & 17.05 \\
\hline Bh 19 & 11.93 & 12.27 & 12.10 & 13.43 & 14.27 & 13.85 \\
\hline Bh 20 & 18.20 & 18.37 & 18.28 & 16.63 & 17.27 & 16.95 \\
\hline Bh 21 & 14.73 & 15.47 & 15.10 & 11.83 & 12.50 & 12.17 \\
\hline Bh 22 & 17.47 & 18.03 & 17.75 & 15.73 & 16.57 & 16.15 \\
\hline Bh 23 & 13.60 & 14.27 & 13.93 & 15.40 & 16.27 & 15.83 \\
\hline Bh 24 & 20.43 & 21.47 & 20.95 & 17.43 & 18.83 & 18.13 \\
\hline Bh 25 & 21.70 & 22.03 & 21.87 & 14.93 & 15.43 & 15.18 \\
\hline Bh 26 & 20.33 & 21.13 & 20.73 & 19.27 & 20.47 & 19.87 \\
\hline Bh 27 & 13.03 & 13.13 & 13.08 & 12.53 & 13.27 & 12.90 \\
\hline $\mathrm{Bh} 28$ & 21.40 & 22.27 & 21.83 & 13.9 & 14.63 & 14.27 \\
\hline Bh 29 & 16.73 & 17.13 & 16.93 & 13.57 & 13.97 & 13.77 \\
\hline $\mathrm{Bh} 30$ & 19.47 & 20.33 & 19.90 & 18.57 & 19.33 & 18.95 \\
\hline $\mathrm{Bh} 31$ & 19.73 & 20.33 & 20.03 & 16.10 & 16.90 & 16.50 \\
\hline Bh 32 & 18.93 & 19.17 & 19.05 & 16.70 & 17.10 & 16.90 \\
\hline Bh 33 & 24.03 & 25.03 & 24.53 & 18.80 & 20.97 & 19.88 \\
\hline Bh 34 & 22.27 & 22.63 & 22.45 & 18.03 & 19.03 & 18.53 \\
\hline Bh 35 & 18.10 & 18.80 & 18.45 & 15.13 & 15.30 & 15.22 \\
\hline Bh 36 & 15.23 & 15.87 & 15.55 & 11.80 & 12.13 & 11.97 \\
\hline Bh 37 & 11.00 & 11.20 & 11.10 & 9.33 & 9.57 & 9.45 \\
\hline Bh 38 & 12.47 & 12.57 & 12.52 & 7.27 & 7.50 & 7.38 \\
\hline Bh 39 & 14.30 & 14.50 & 14.40 & 9.87 & 10.07 & 9.97 \\
\hline $\mathrm{Bh} 40$ & 13.03 & 13.33 & 13.18 & 9.83 & 10.30 & 10.07 \\
\hline Mean & 18.36 & 18.93 & 18.64 & 15.45 & 16.25 & 15.85 \\
\hline Range & $26.33-11.00$ & $27.40-11.20$ & $26.87-11.10$ & $24.63-7.27$ & $24.70-7.50$ & $24.67-7.38$ \\
\hline $\mathrm{CV}$ & 7.65 & 7.67 & 7.61 & 6.68 & 7.73 & 6.90 \\
\hline SEd & 0.81 & 0.84 & 1.16 & 0.59 & 0.73 & 0.89 \\
\hline $\mathrm{CD}(\mathrm{P}=0.05)$ & 2.28 & 2.36 & 2.31 & 1.67 & 2.04 & 1.78 \\
\hline
\end{tabular}


Table 9: Per Se Performance of Ash Gourd Genotypes for Number of Seeds per Fruit and Hundred-Seed Weight during Kharif, Summer and Pooled Data

\begin{tabular}{|c|c|c|c|c|c|c|}
\hline \multirow[b]{2}{*}{ Acc. No. } & \multicolumn{3}{|c|}{ Number of Seeds Per Fruit } & \multicolumn{3}{|c|}{ Hundred-Seed Weight (g) } \\
\hline & Kharif & Summer & Pooled & Kharif & Summer & Pooled \\
\hline Bh 1 & 505.87 & 541.39 & 523.63 & 7.17 & 7.59 & 7.38 \\
\hline Bh 2 & 531.93 & 576.16 & 554.05 & 7.99 & 8.37 & 8.18 \\
\hline $\mathrm{Bh} 3$ & 367.85 & 378.97 & 373.41 & 7.73 & 8.21 & 7.97 \\
\hline Bh 4 & 557.08 & 602.11 & 579.59 & 9.36 & 9.72 & 9.54 \\
\hline Bh 5 & 994.10 & 1055.79 & 1024.95 & 7.30 & 7.55 & 7.43 \\
\hline Bh 6 & 649.68 & 693.05 & 671.37 & 7.69 & 8.03 & 7.86 \\
\hline Bh 7 & 589.60 & 638.72 & 614.17 & 7.65 & 7.93 & 7.79 \\
\hline Bh 8 & 312.51 & 351.02 & 331.77 & 9.14 & 9.56 & 9.35 \\
\hline Bh 9 & 1040.10 & 1111.88 & 1075.99 & 8.95 & 9.36 & 9.15 \\
\hline Bh 10 & 631.61 & 684.17 & 657.89 & 7.17 & 7.50 & 7.34 \\
\hline Bh 11 & 631.68 & 684.74 & 658.21 & 8.62 & 9.05 & 8.84 \\
\hline Bh 12 & 510.41 & 537.06 & 523.74 & 8.23 & 8.66 & 8.44 \\
\hline Bh 13 & 249.11 & 264.61 & 256.86 & 8.67 & 9.38 & 9.02 \\
\hline Bh 14 & 604.96 & 651.36 & 628.16 & 9.74 & 10.11 & 9.93 \\
\hline Bh 15 & 555.66 & 587.40 & 571.53 & 8.53 & 9.06 & 8.79 \\
\hline Bh 16 & 548.34 & 595.80 & 572.08 & 9.31 & 9.67 & 9.49 \\
\hline Bh 17 & 693.93 & 755.74 & 724.84 & 9.81 & 10.64 & 10.23 \\
\hline Bh 18 & 448.77 & 483.83 & 466.30 & 7.89 & 8.27 & 8.08 \\
\hline Bh 19 & 257.00 & 279.77 & 268.39 & 9.35 & 10.01 & 9.68 \\
\hline Bh 20 & 775.24 & 837.08 & 806.16 & 9.09 & 9.84 & 9.47 \\
\hline Bh 21 & 494.55 & 524.34 & 509.45 & 9.60 & 10.02 & 9.81 \\
\hline Bh 22 & 553.69 & 591.52 & 572.61 & 8.52 & 8.92 & 8.72 \\
\hline Bh 23 & 582.83 & 627.26 & 605.05 & 8.86 & 9.25 & 9.05 \\
\hline Bh 24 & 452.36 & 480.38 & 466.37 & 7.94 & 8.45 & 8.20 \\
\hline Bh 25 & 517.68 & 559.58 & 538.63 & 7.29 & 7.69 & 7.49 \\
\hline Bh 26 & 594.31 & 629.23 & 611.77 & 7.53 & 8.16 & 7.85 \\
\hline Bh 27 & 363.53 & 384.55 & 374.04 & 7.82 & 8.23 & 8.02 \\
\hline Bh 28 & 358.72 & 376.62 & 367.67 & 7.14 & 7.55 & 7.35 \\
\hline Bh 29 & 483.32 & 518.82 & 501.08 & 8.60 & 8.99 & 8.79 \\
\hline Bh 30 & 671.09 & 704.93 & 688.01 & 9.59 & 10.22 & 9.91 \\
\hline Bh 31 & 491.63 & 523.71 & 507.67 & 9.12 & 9.41 & 9.26 \\
\hline Bh 32 & 367.76 & 386.80 & 377.28 & 8.64 & 9.29 & 8.97 \\
\hline Bh 33 & 783.25 & 858.16 & 820.71 & 8.62 & 9.04 & 8.83 \\
\hline Bh 34 & 157.36 & 169.59 & 163.48 & 7.38 & 7.69 & 7.53 \\
\hline Bh 35 & 267.72 & 289.39 & 278.56 & 10.01 & 10.46 & 10.23 \\
\hline Bh 36 & 342.34 & 361.05 & 351.70 & 8.15 & 8.72 & 8.43 \\
\hline Bh 37 & 156.94 & 170.59 & 163.77 & 7.23 & 7.90 & 7.56 \\
\hline Bh 38 & 204.93 & 214.77 & 209.85 & 6.43 & 6.77 & 6.60 \\
\hline Bh 39 & 296.03 & 310.35 & 303.19 & 5.80 & 6.17 & 5.99 \\
\hline Bh 40 & 114.87 & 124.87 & 119.87 & 5.98 & 6.42 & 6.20 \\
\hline Mean & 492.76 & 527.93 & 510.35 & 8.24 & 8.70 & 8.47 \\
\hline Range & $\begin{array}{l}1040.10 \\
-114.87\end{array}$ & $\begin{array}{l}111.88- \\
124.87 \\
\end{array}$ & $\begin{array}{l}1075.99 \\
-119.87 \\
\end{array}$ & $\begin{array}{l}10.01- \\
5.80\end{array}$ & $\begin{array}{l}10.64- \\
6.17 \\
\end{array}$ & $\begin{array}{l}10.23- \\
5.99 \\
\end{array}$ \\
\hline CV & 2.26 & 4.62 & 2.99 & 8.83 & 9.12 & 4.54 \\
\hline SEd & 6.44 & 14.09 & 12.47 & 0.42 & 0.46 & 0.31 \\
\hline $\mathrm{CD}(\mathrm{P}=0.05)$ & 18.14 & 39.68 & 24.83 & 1.18 & 1.29 & 0.63 \\
\hline
\end{tabular}


Table 10: Per se Performance of Ash Gourd Genotypes for Number of Fruits per Vine and Yield per Vine during Kharif, Summer and Pooled Data

\begin{tabular}{|c|c|c|c|c|c|c|}
\hline \multirow[b]{2}{*}{ Acc. No. } & \multicolumn{3}{|c|}{ Number of Fruits Per Vine } & \multicolumn{3}{|c|}{ Yield Per Vine (kg) } \\
\hline & Kharif & Summer & Pooled & Kharif & Summer & Pooled \\
\hline $\mathrm{Bh} 1$ & 2.55 & 2.94 & 2.74 & 5.27 & 6.64 & 5.96 \\
\hline $\mathrm{Bh} 2$ & 2.98 & 3.45 & 3.21 & 5.29 & 6.75 & 6.02 \\
\hline Bh 3 & 2.46 & 3.40 & 2.93 & 4.94 & 6.30 & 5.62 \\
\hline $\mathrm{Bh} 4$ & 2.63 & 3.04 & 2.83 & 7.18 & 8.95 & 8.07 \\
\hline Bh 5 & 2.08 & 2.63 & 2.35 & 6.37 & 8.11 & 7.24 \\
\hline Bh 6 & 2.02 & 2.33 & 2.17 & 5.37 & 6.84 & 6.11 \\
\hline $\mathrm{Bh} 7$ & 2.30 & 3.02 & 2.66 & 14.37 & 18.51 & 16.44 \\
\hline $\mathrm{Bh} 8$ & 2.13 & 2.46 & 2.29 & 5.46 & 5.77 & 5.62 \\
\hline Bh 9 & 2.16 & 2.49 & 2.32 & 4.28 & 6.21 & 5.24 \\
\hline $\mathrm{Bh} 10$ & 2.71 & 3.14 & 2.92 & 6.19 & 7.73 & 6.96 \\
\hline $\mathrm{Bh} 11$ & 2.25 & 2.61 & 2.43 & 5.06 & 7.61 & 6.34 \\
\hline Bh 12 & 2.25 & 2.83 & 2.54 & 5.73 & 10.03 & 7.88 \\
\hline Bh 13 & 2.38 & 2.74 & 2.56 & 4.11 & 5.19 & 4.65 \\
\hline $\mathrm{Bh} 14$ & 2.38 & 2.75 & 2.57 & 4.55 & 6.15 & 5.36 \\
\hline Bh 15 & 2.55 & 2.95 & 2.75 & 3.72 & 4.57 & 4.15 \\
\hline Bh 16 & 2.08 & 2.42 & 2.25 & 8.12 & 10.31 & 9.22 \\
\hline Bh 17 & 2.21 & 2.56 & 2.38 & 4.05 & 5.13 & 4.59 \\
\hline Bh 18 & 2.96 & 3.44 & 3.2 & 6.72 & 8.83 & 7.78 \\
\hline Bh 19 & 3.39 & 3.93 & 3.66 & 14.39 & 18.17 & 16.28 \\
\hline $\mathrm{Bh} 20$ & 2.38 & 3.16 & 2.77 & 6.52 & 8.26 & 7.39 \\
\hline $\mathrm{Bh} 21$ & 2.55 & 2.95 & 2.75 & 4.79 & 5.93 & 5.37 \\
\hline $\mathrm{Bh} 22$ & 3.84 & 4.45 & 4.14 & 12.61 & 15.95 & 14.29 \\
\hline Bh 23 & 2.46 & 2.85 & 2.65 & 4.94 & 6.25 & 5.60 \\
\hline $\mathrm{Bh} 24$ & 2.81 & 3.26 & 3.04 & 6.98 & 8.75 & 7.87 \\
\hline $\mathrm{Bh} 25$ & 1.96 & 2.27 & 2.11 & 4.96 & 6.28 & 5.62 \\
\hline Bh 26 & 2.25 & 3.17 & 2.71 & 4.49 & 5.66 & 5.07 \\
\hline Bh 27 & 3.03 & 3.51 & 3.27 & 7.26 & 9.18 & 8.22 \\
\hline $\mathrm{Bh} 28$ & 2.30 & 2.99 & 2.64 & 6.40 & 8.32 & 7.37 \\
\hline Bh 29 & 2.72 & 3.16 & 2.94 & 5.85 & 7.43 & 6.64 \\
\hline $\mathrm{Bh} 30$ & 3.25 & 3.77 & 3.51 & 10.36 & 12.34 & 11.35 \\
\hline $\mathrm{Bh} 31$ & 3.02 & 3.50 & 3.26 & 4.19 & 5.30 & 4.74 \\
\hline $\mathrm{Bh} 32$ & 2.72 & 3.16 & 2.94 & 6.36 & 8.06 & 7.21 \\
\hline $\mathrm{Bh} 33$ & 2.38 & 2.75 & 2.56 & 5.65 & 6.94 & 6.30 \\
\hline $\mathrm{Bh} 34$ & 2.25 & 4.70 & 3.47 & 7.35 & 9.92 & 8.64 \\
\hline Bh 35 & 2.08 & 2.41 & 2.24 & 2.95 & 3.98 & 3.47 \\
\hline Bh 36 & 2.25 & 2.94 & 2.59 & 5.43 & 6.92 & 6.18 \\
\hline $\mathrm{Bh} 37$ & 1.92 & 2.22 & 2.06 & 1.17 & 1.47 & 1.32 \\
\hline Bh 38 & 2.42 & 2.79 & 2.61 & 0.89 & 1.37 & 1.13 \\
\hline Bh 39 & 1.96 & 2.27 & 2.11 & 1.13 & 1.64 & 1.39 \\
\hline $\mathrm{Bh} 40$ & 2.25 & 2.60 & 2.43 & 0.77 & 1.06 & 0.92 \\
\hline Mean & 2.48 & 3.00 & 2.74 & 5.81 & 7.47 & 6.64 \\
\hline Range & $3.84-1.92$ & $4.70-2.22$ & $4.14-2.06$ & $14.39-0.77$ & $18.51-1.06$ & $16.44-0.92$ \\
\hline $\mathrm{CV}$ & 13.96 & 6.59 & 10.65 & 6.19 & 4.03 & 7.97 \\
\hline SEd & 0.2 & 0.29 & 0.24 & 0.21 & 0.17 & 0.21 \\
\hline $\mathrm{CD}(\mathrm{P}=0.05)$ & 0.56 & 0.81 & 0.46 & 0.58 & 0.49 & 0.42 \\
\hline
\end{tabular}


Table 11: Per se Performance of Ash Gourd Genotypes for Carbohydrate Content and Crude Fibre Content during Kharif, Summer and Pooled Data

\begin{tabular}{|c|c|c|c|c|c|c|}
\hline \multirow[b]{2}{*}{ Acc. No. } & \multicolumn{3}{|c|}{ Carbohydrate Content (mg/100g) } & \multicolumn{3}{|c|}{ Crude Fibre content (\%) } \\
\hline & Kharif & Summer & Pooled & Kharif & Summer & Pooled \\
\hline Bh 1 & 3.52 & 3.62 & 3.57 & 0.34 & 0.33 & 0.34 \\
\hline $\mathrm{Bh} 2$ & 3.22 & 3.31 & 3.27 & 0.62 & 0.69 & 0.66 \\
\hline $\mathrm{Bh} 3$ & 3.14 & 3.23 & 3.19 & 0.49 & 0.48 & 0.49 \\
\hline $\mathrm{Bh} 4$ & 3.47 & 3.56 & 3.51 & 0.49 & 0.46 & 0.48 \\
\hline Bh 5 & 4.51 & 4.63 & 4.57 & 0.51 & 0.54 & 0.53 \\
\hline Bh 6 & 3.15 & 3.22 & 3.19 & 0.62 & 0.74 & 0.68 \\
\hline $\mathrm{Bh} 7$ & 3.31 & 3.4 & 3.36 & 0.54 & 0.51 & 0.53 \\
\hline $\mathrm{Bh} 8$ & 3.90 & 4.03 & 3.97 & 0.49 & 0.64 & 0.57 \\
\hline Bh 9 & 3.22 & 3.30 & 3.26 & 0.48 & 0.6 & 0.54 \\
\hline Bh 10 & 0.89 & 0.91 & 0.9 & 0.51 & 0.47 & 0.49 \\
\hline Bh 11 & 3.23 & 3.32 & 3.28 & 0.49 & 0.45 & 0.47 \\
\hline Bh 12 & 4.99 & 5.12 & 5.06 & 0.48 & 0.43 & 0.46 \\
\hline Bh 13 & 6.11 & 6.29 & 6.20 & 0.48 & 0.50 & 0.49 \\
\hline Bh 14 & 3.26 & 3.35 & 3.31 & 0.43 & 0.43 & 0.43 \\
\hline Bh 15 & 4.91 & 5.06 & 4.99 & 0.42 & 0.45 & 0.44 \\
\hline Bh 16 & 5.04 & 5.19 & 5.11 & 0.40 & 0.37 & 0.38 \\
\hline Bh 17 & 4.49 & 4.62 & 4.56 & 0.38 & 0.37 & 0.38 \\
\hline Bh 18 & 5.42 & 5.59 & 5.51 & 0.42 & 0.45 & 0.44 \\
\hline Bh 19 & 3.40 & 3.51 & 3.46 & 0.41 & 0.36 & 0.39 \\
\hline $\mathrm{Bh} 20$ & 3.31 & 3.40 & 3.36 & 0.40 & 0.45 & 0.43 \\
\hline Bh 21 & 3.58 & 3.69 & 3.64 & 0.41 & 0.46 & 0.44 \\
\hline $\mathrm{Bh} 22$ & 3.77 & 3.88 & 3.83 & 0.44 & 0.49 & 0.47 \\
\hline Bh 23 & 4.40 & 4.53 & 4.47 & 0.44 & 0.47 & 0.46 \\
\hline Bh 24 & 5.59 & 5.75 & 5.67 & 0.51 & 0.56 & 0.53 \\
\hline $\mathrm{Bh} 25$ & 3.08 & 3.18 & 3.13 & 0.44 & 0.40 & 0.42 \\
\hline Bh 26 & 4.42 & 4.54 & 4.48 & 0.46 & 0.52 & 0.49 \\
\hline Bh 27 & 4.99 & 5.14 & 5.07 & 0.52 & 0.51 & 0.52 \\
\hline $\mathrm{Bh} 28$ & 3.89 & 4.00 & 3.95 & 0.54 & 0.52 & 0.53 \\
\hline Bh 29 & 3.61 & 3.72 & 3.67 & 0.48 & 0.44 & 0.46 \\
\hline Bh 30 & 5.10 & 5.24 & 5.17 & 0.49 & 0.49 & 0.49 \\
\hline Bh 31 & 3.35 & 3.44 & 3.39 & 0.48 & 0.41 & 0.45 \\
\hline Bh 32 & 5.04 & 5.19 & 5.12 & 0.62 & 0.55 & 0.59 \\
\hline Bh 33 & 4.96 & 5.12 & 5.05 & 0.58 & 0.53 & 0.56 \\
\hline Bh 34 & 3.61 & 3.71 & 3.66 & 0.49 & 0.43 & 0.46 \\
\hline Bh 35 & 3.86 & 3.97 & 3.92 & 0.49 & 0.39 & 0.44 \\
\hline Bh 36 & 5.02 & 5.16 & 5.10 & 0.48 & 0.44 & 0.46 \\
\hline Bh 37 & 5.45 & 5.62 & 5.54 & 0.44 & 0.47 & 0.46 \\
\hline Bh 38 & 5.47 & 5.61 & 5.54 & 0.38 & 0.36 & 0.37 \\
\hline Bh 39 & 6.10 & 6.28 & 6.19 & 0.37 & 0.34 & 0.36 \\
\hline $\mathrm{Bh} 40$ & 3.67 & 3.77 & 3.73 & 0.38 & 0.39 & 0.39 \\
\hline Mean & 4.14 & 4.25 & 4.20 & 0.47 & 0.47 & 0.47 \\
\hline Range & $6.11-0.89$ & $6.29-0.91$ & $6.20-0.90$ & $0.62-0.34$ & $0.74-0.33$ & $0.68-0.34$ \\
\hline $\mathrm{CV}$ & 7.85 & 8.09 & 6.51 & 10.63 & 8.74 & 5.13 \\
\hline SEd & 0.19 & 0.19 & 0.27 & 0.03 & 0.02 & 0.23 \\
\hline $\mathrm{CD}(\mathrm{P}=0.05)$ & 0.53 & 0.56 & 0.54 & 0.08 & 0.07 & 0.05 \\
\hline
\end{tabular}


Table 12: Per se Performance of Ash Gourd Genotypes for Protein Content and Total Soluble Solids during Kharif, Summer and Pooled Data

\begin{tabular}{|c|c|c|c|c|c|c|}
\hline \multirow[b]{2}{*}{ Acc. No. } & \multicolumn{3}{|c|}{ Protein Content (mg/100g) } & \multicolumn{3}{|c|}{ Total Soluble Solids ( ${ }^{0}$ Brix) } \\
\hline & Kharif & Summer & Pooled & Kharif & Summer & Pooled \\
\hline $\mathrm{Bh} 1$ & 350.43 & 369.01 & 359.72 & 2.50 & 2.60 & 2.55 \\
\hline Bh 2 & 311.75 & 317.26 & 314.51 & 3.27 & 3.37 & 3.32 \\
\hline $\mathrm{Bh} 3$ & 384.79 & 389.3 & 387.05 & 1.27 & 1.37 & 1.32 \\
\hline $\mathrm{Bh} 4$ & 301.90 & 313.09 & 307.50 & 1.00 & 1.10 & 1.05 \\
\hline Bh 5 & 447.77 & 437.55 & 442.66 & 2.70 & 2.80 & 2.75 \\
\hline Bh 6 & 305.52 & 320.47 & 313.00 & 1.80 & 1.90 & 1.85 \\
\hline Bh 7 & 324.54 & 324.33 & 324.43 & 0.30 & 0.40 & 0.35 \\
\hline Bh 8 & 340.96 & 347.99 & 344.48 & 2.20 & 2.30 & 2.25 \\
\hline Bh 9 & 403.64 & 408.92 & 406.28 & 1.57 & 1.90 & 1.73 \\
\hline $\mathrm{Bh} 10$ & 250.58 & 260.38 & 255.48 & 2.40 & 2.50 & 2.45 \\
\hline $\mathrm{Bh} 11$ & 250.94 & 261.13 & 256.04 & 1.13 & 1.23 & 1.18 \\
\hline Bh 12 & 262.32 & 271.65 & 266.99 & 0.97 & 1.40 & 1.18 \\
\hline Bh 13 & 277.52 & 285.21 & 281.37 & 2.87 & 2.97 & 2.92 \\
\hline Bh 14 & 222.49 & 223.31 & 222.9 & 2.07 & 2.17 & 2.12 \\
\hline Bh 15 & 260.41 & 269.24 & 264.83 & 2.17 & 2.27 & 2.22 \\
\hline Bh 16 & 391.94 & 363.02 & 377.48 & 2.03 & 2.13 & 2.08 \\
\hline Bh 17 & 310.40 & 327.47 & 318.94 & 0.50 & 1.20 & 0.85 \\
\hline Bh 18 & 322.66 & 226.09 & 274.38 & 3.73 & 3.83 & 3.78 \\
\hline Bh 19 & 311.74 & 322.63 & 317.19 & 2.47 & 2.57 & 2.52 \\
\hline $\mathrm{Bh} 20$ & 330.54 & 325.57 & 328.05 & 1.30 & 1.40 & 1.35 \\
\hline $\mathrm{Bh} 21$ & 285.28 & 291.06 & 288.17 & 2.27 & 2.37 & 2.32 \\
\hline $\mathrm{Bh} 22$ & 293.93 & 313.26 & 303.6 & 1.07 & 1.70 & 1.38 \\
\hline Bh 23 & 335.85 & 334.38 & 335.12 & 1.80 & 1.83 & 1.82 \\
\hline $\mathrm{Bh} 24$ & 333.7 & 335.99 & 334.85 & 1.97 & 2.30 & 2.13 \\
\hline $\mathrm{Bh} 25$ & 350.44 & 354.11 & 352.27 & 2.87 & 2.43 & 2.65 \\
\hline Bh 26 & 264.14 & 275.42 & 269.78 & 2.53 & 2.43 & 2.48 \\
\hline Bh 27 & 310.49 & 335.89 & 323.19 & 3.03 & 2.50 & 2.77 \\
\hline $\mathrm{Bh} 28$ & 317.69 & 320.06 & 318.88 & 3.23 & 3.23 & 3.23 \\
\hline Bh 29 & 323.67 & 323.4 & 323.54 & 2.47 & 2.67 & 2.57 \\
\hline $\mathrm{Bh} 30$ & 299.42 & 304.23 & 301.82 & 4.27 & 4.13 & 4.20 \\
\hline $\mathrm{Bh} 31$ & 269.37 & 273.54 & 271.46 & 2.50 & 2.93 & 2.72 \\
\hline Bh 32 & 340.60 & 353.67 & 347.14 & 3.40 & 3.50 & 3.45 \\
\hline $\mathrm{Bh} 33$ & 334.97 & 259.56 & 297.27 & 0.83 & 1.27 & 1.05 \\
\hline $\mathrm{Bh} 34$ & 353.00 & 361.15 & 357.08 & 3.53 & 3.63 & 3.58 \\
\hline $\mathrm{Bh} 35$ & 378.08 & 402.42 & 390.25 & 3.23 & 3.33 & 3.28 \\
\hline $\mathrm{Bh} 36$ & 272.29 & 284.21 & 278.25 & 2.83 & 2.93 & 2.88 \\
\hline $\mathrm{Bh} 37$ & 281.57 & 288.61 & 285.09 & 5.73 & 5.83 & 5.78 \\
\hline $\mathrm{Bh} 38$ & 315.77 & 315.84 & 315.81 & 6.43 & 6.53 & 6.48 \\
\hline $\mathrm{Bh} 39$ & 313.12 & 330.50 & 321.81 & 4.27 & 4.37 & 4.32 \\
\hline $\mathrm{Bh} 40$ & 349.76 & 351.89 & 350.83 & 5.20 & 5.30 & 5.25 \\
\hline Mean & 317.15 & 319.32 & 318.24 & 2.54 & 2.67 & 2.60 \\
\hline Range & $447.77-222.49$ & $437.55-223.31$ & $442.66-222.90$ & $6.43-0.30$ & $6.53-0.40$ & $6.48-0.35$ \\
\hline $\mathbf{C V}$ & 3.45 & 9.09 & 12.93 & 13.84 & 15.70 & 3.84 \\
\hline SEd & 6.32 & 16.76 & 13.35 & 0.2 & 0.24 & 0.28 \\
\hline $\mathrm{CD}(\mathrm{P}=0.05)$ & 17.80 & 47.19 & 26.57 & 0.57 & 0.68 & 0.55 \\
\hline
\end{tabular}

